\title{
Use of an Amplatzer ductal occluder to close a persistent left superior caval vein which reopened after a total cavopulmonary anastomosis
}

\author{
Marie-Chri stine Seghaye, Uwe Wainwright, ${ }^{1}$ Götz von Bernuth \\ Department of Paed iatric Cardiology and ${ }^{1}$ Department of Anesthesiology, Aachen University of Technology, \\ Aachen, Germany
}

\begin{abstract}
A 7-year-old boy devel oped increasing cyanosis after a total cavopulmonary connection with a $3 \mathrm{~mm}$ fenestration in the baffle. Catheterisation performed 4 years and 7 months after the operation showed reopening of a left superior caval vein draining into the pulmonary venous atrium. Due to the large size of the left superior caval vein, and the absence of intrinsic stenosis, we chose to use an Amplatzer ductal device to occlude the reopened vein. The procedure was safe and successful.
\end{abstract}

Keywords: Total cavopulmonary connection; reopening left superior caval vein; Amplatzer duct occluder

$\mathrm{R}$

EOPENING OF A LEFT SUPERIOR CAVAL VEIN CAN occur early or late after the creation of cavopulmonary connections. The reopening can cause profound cyanosis due to veno-venous rightto-left shunting. ${ }^{1,2}$ If the reopened left superior caval vein is small, or has intrinsic stenosis, embolisation using coils is considered the treatment of choice. ${ }^{3,4}$ In the case we report here, due to the large size of the venous channel and the absence of stenosis, we closed the vein using an Amplatzer ductal occluder.

\section{Case report}

The patient was a 7-year-old boy with usual atrial arrangement, tricuspid atresia with discordant ventriculo-arterial connections, a restrictive ventricular septal defect, and coarctation of the aorta with a hypoplastic aortic arch and right descending aorta. Repair of the aortic arch had been performed at the age of 8 weeks, followed by a Damus-Kaye-Stansel

Correspondence to: Marie-Christine Seghaye MD, Department of Paediatric Cardiology, German Heart Centre Munich, Lazarettstrasse 36, D-80636 Munich, Germany. Tel: +49 891218 3011; Fax: +49 891218 3013; E-mail: seghaye@dhm.mhn.de

Accepted for publication 19 October 2001 anastomosis, atrioseptectomy and construction of a central aorto-pulmonary anastomosis at the age of 10 weeks, and a total cavopulmonary connection at the age of 2 years and 5 months, respectively. A $3 \mathrm{~mm}$ fenestration was created in the baffle. Persistence of the left superior caval vein had been excluded by angiography prior to the total cavopulmonary connection. The saturation of oxygen measured transcutaneously at discharge after the total cavopulmonary connection was $95 \%$. The patient was then readmitted for hemodynamic and anatomic evaluation at the age of 7 years because of increasing cyanosis.

Clinical findings at admission revealed a good general state, but cyanosis was noted, with an oxygen saturation of $82 \%$ at rest and of $79 \%$ during submaximal exercise. Transoesophageal echocardiography showed a small fenestration of the caval venous tunnel, measuring $2.5-3 \mathrm{~mm}$, which permitted a right-to-left shunt.

Catheterisation was performed under general anaesthesia and continuous infusion of heparin at $10,000 \mathrm{IU} / \mathrm{m}^{2} /$ day. The haemodynamics were satisfactory, with a moderately increased central venous pressure of $12 \mathrm{mmHg}$. The total cavopulmonary connection was unrestricted, and the pulmonary occlusion pressure was $8 \mathrm{mmHg}$. Under mechanical ventilation, and at an inspired oxygen fraction of 0.30 , mixed 

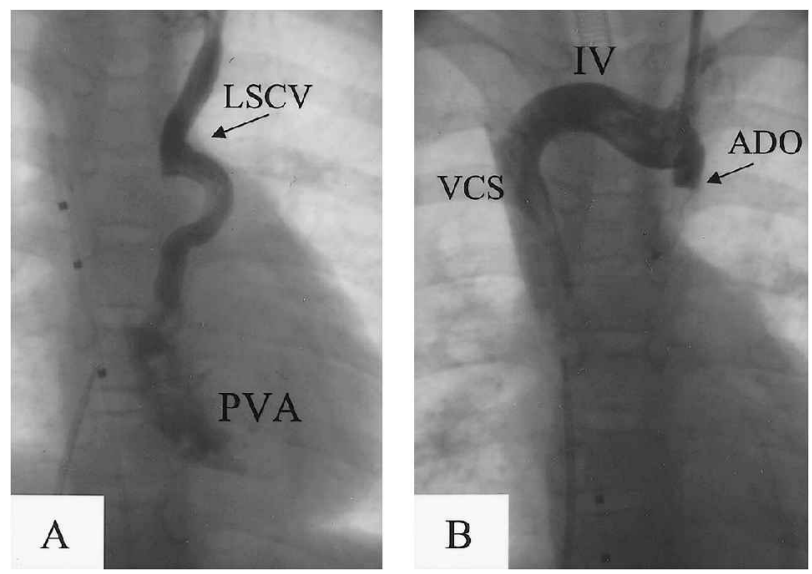

Figure 1.

(A) Selective ang iog ram into the reopened left superior caval vein (LSCV) draining into the pulmonary venous atrium (PVA). (B) Selective angiog ram in the proximal part of the left superior caval vein obstructed with the Amplatzer duct occluder (ADO). IV: brachiocephal ic vein; VCS: superior caval vein.

venous oxygen saturation was $70 \%$, aortic oxygen saturation 98\%, and aortic partial oxygen pressure 120 Torr. Angiography showed an unobstructed cavopulmonary connection, an adequate DamusKaye-Stansel anastomosis without arterial valvar insufficiency, a small fenestration in the caval venous baffle, and a large reopened left superior caval vein, diameter $7 \mathrm{~mm}$, without significant intrinsic stenosis, draining into the pulmonary venous atrium (Fig. 1A).

After catheterisation of the left internal jugular vein, a $7 F / 100 \mathrm{~cm}$ long Gensini catheter was introduced through a 7 French sheath over a wire. The Gensini catheter served as long sheath, allowing the introduction of the delivery system for an Amplatzer ductal occluder connected to a $8 / 10 \mathrm{~mm}$ device. This technique was adopted due to difficulty with introduction of the terminal curve of the Amplatzer long sheath into the left superior caval vein. After verification of an adequate position for the device by injection of contrast medium into the proximal part of the left superior caval vein, the occluder was disconnected from its delivery system. Angiography showed complete occlusion of the left superi or caval vein, and unrestricted flow through the brachiocephalic vein (Fig. 1B). Caval venous pressure, mixed venous oxygen saturation, and aortic oxygen saturation remained unchanged after the procedure, but aortic partial oxygen pressure rose to 334 Torr. After extubation, arterial oxygen saturation was $96 \%$ in room air.

Recovery was uncomplicated. Chest radiography performed $48 \mathrm{~h}$ after the procedure showed an unchanged position of the device. The patient was discharged $72 \mathrm{~h}$ after the catheterisation in good clinical condition and without cyanosis.

\section{Discussion}

Persistence of the left superior caval vein is present in up to $0.4 \%$ of the normal population, and in up to $10 \%$ of patients with congenital cardiac defects. ${ }^{1}$ The anomaly is thought to be the result of reduced compression of the left-sided cardinal vein between the left atrium and the hilum of the left lung during embryological life. ${ }^{1}$

Reopening of a left superior caval vein is a not uncommon complication early or late after construction of cavopulmonary connections, leading to reduced pulmonary blood flow and increasing clinical cyanosis due to a veno-venous right-to-left shunt. ${ }^{2,5}$ Postoperative abnormal hemodynamics, with an increased pressure gradient between the systemic and pulmonary venous system, could be responsible for this feature..$^{5}$

In our patient, as with previous reports, the left superior caval vein was not patent before construction of the total cavopulmonary connection, but obviously was not obliterated since it reopened during the postoperative course. This led to cyanosis at rest which increased with exercise, giving the indication for treatment.

Therapeutic strategies for the closure of a reopened left superior caval vein are either surgical ligation ${ }^{6}$ or transcatheter closure. Coil embolisation has been successfully performed after a bidirectional cavopulmonary anastomosis. ${ }^{3,4}$ In our patient, the vein was unsuitable for coil embolisation due to its large size, its connection with the pulmonary venous atrium, and the absence of intrinsic stenosis.

The Amplatzer ductal occluder, originally designed for the closure of the patent arterial duct, consists of a self-expanding nitinol mesh which, when deployed, results in stenting of the vessel and compression of its wall, ensuring positional stability. ${ }^{7}$ Due to these properties, the occluder appeared to be a reasonable choice for the closure of the left superior caval vein in our patient. By placing a large sized occluder proximal to the junction of the vein with the pulmonary venous atrium, we attained complete closure, also achieving positional stability of the device.

Due to its proximal curvature, the Amplatzer long sheath could not be introduced into the left superior caval vein, leading us to utilise a Gensini catheter to introduce the Amplatzer delivery system. This allowed adequate positioning of the device, and injections of contrast medium for the control of occlusion after deployment of the occluder. This technique was safe and successful. This is, to the best of our knowledge, the first report on closure of 
a reopened left superior caval vein with an Amplatzer ductal occluder.

\section{References}

1. Nsah EN, Moore GW, Hutchins GM. Pathogenesis of persistent left superior vena cava with a coronary sinus connection. Pediatr Pathol 1991; 11: 261-269.

2. Filippini LH, Ovaert C, Nykanen DG, Freedom RM. Reopening of persistent left superior caval vein after bidirectional cavopulmonary connections. Heart 1998; 79: 509-512.

3. Michel-Behnke I, Akinturk H, Schranz D. Reopening of a persistent left superior vena cava in the early postoperative period following bidirectional cavopulmonary anastomosis - treatment by coil embolization. Z Kardiol 1999; 88: 555-558.

4. Stümper O, Wright JG, Sadiq M, De Giovanni JV. Late systemic desaturation after total cavopulmonary shunt operations. Br Heart J 1995; 74: 282-286.

5. Magee AG, McCrindle BW, Mawson J, Benson LN, Williams WG, Freedom RM. Systemic venous collateral development after the bidirectional cavopulmonary anastomosis. Prevalence and predictors. J Am Coll Cardiol 1998; 32: 502-508.

6. Reddy VM, McElhinney DB, Hanley FL. Correction of left superior vena cava draining to the left atrium using extracardiac techniques. Ann Thorac Surg 1997; 63: 1800-1802.

7. Bilkis AA, Alwi M, Hasri S, et al. The Amplatzer duct occluder: experience in 209 patients. J Am Coll Cardiol 2001; 37: 258-261. 